\title{
Challenges of Anticoagulation in Total Joint Arthroplasty
}

\author{
Anshul Sobti*, Ashwin Unnithan \\ FRCS (Tr \& Orth), Rowley Bristow Unit, Ashford and St. Peter's Hospitals NHS Foundation Trust, Surrey, UK \\ Email: ^anshulsobti@nhs.net
}

How to cite this paper: Sobti, A. and Unnithan, A. (2020) Challenges of Anticoagulation in Total Joint Arthroplasty. Open Journal of Orthopedics, 10, 289-294. https://doi.org/10.4236/ojo.2020.1010029

Received: September 28, 2020

Accepted: October 19, 2020

Published: October 22, 2020

Copyright $\odot 2020$ by author(s) and Scientific Research Publishing Inc. This work is licensed under the Creative Commons Attribution International License (CC BY 4.0).

http://creativecommons.org/licenses/by/4.0/

\begin{abstract}
Aggressive anticoagulation following total joint arthroplaty (TJA) is well known to increase the risk of both wound leakage and hematoma formation. Despite this there are no guidelines for orthopaedic surgeons undertaking major primary and revision surgery in patients with high risk of venous thromboembolic events, who may present with complications directly linked to anticoagulant use. Hence the authors felt the need for this narrative review. They have tried to answer few questions that are commonly faced in clinical practice. Balanced anticoagulation is a difficult task to achieve. The potential consequences of over anticoagulation on the outcome of TJA and revision TJA are live changing and devastating. On the contrary falling short of adequate anticoagulation is fatal leading to recurrent thromboembolic events. Robust risk stratification tools and a multidisciplinary approach are certainly the way forward. There is an urgent need for specialty specific guidelines for managing patient on anticoagulation undergoing TJA, and revision arthroplasty procedures.
\end{abstract}

\section{Keywords}

DVT, Anticoagulation, VTE, Total Joint Arthroplasty, TJA, Revision Arthroplasty

\section{Introduction}

Total joint arthroplasty (TJA) is well associated with increased risk of venous thromboembolic events (VTE) [1] [2] [3]. Various prophylactic methods have been used to minimize this risk, these include intra-operative steps, use of suitable anesthesia, use of pharmacological and non-pharmacological devices etc [4]. Despite all these measures being successful in reducing the risk of VTE, their use is not without risk [4]. Various aspects control the choice of anticoagulation; 
these could be patient factors, surgical procedure factors, risk vs. benefit factors, risk stratification, feasibility and cost related [1] [2] [3] [5] [6] [7].

Prosthetic joint infection (PJI) following TJA is a devastating complication leading to prolonged hospital stay, high mortality and morbidity, suboptimal functional outcome and high social burden [8]. Of the various causes, persistent wound leakage is an important contributing factor as it may provide a portal of entry for pathogens and indicate an underlying haematoma [9]. Aggressive anticoagulation following TJA is well known to increase the risk of both wound leakage and hematoma formation [2]. Each day of prolonged wound leakage increases the risk of wound infection by $42 \%$ following total hip arthoplasty [10]. Patients who require revision arthroplasty due to infection often have a number of co-morbidities and are more likely to be on therapeutic anticoagulation. Despite this there are no guidelines for orthopaedic surgeons undertaking major revision surgery in patients at high risk of VTE, who may present due to complications directly linked to anticoagulant use. Hence the authors felt the need for this narrative review. The authors have tried to answer the following questions that are commonly faced in clinical practice: 1) Is over anticoagulation a problem; 2) What is the best guidance on use of anticoagulant to prevent and treat VTE; 3) Which anticoagulant drug works best; 4) What is the risk of recurrence; 5) Is recurrence of VTE time based post-operatively? What is the ideal duration of treatment, 6) Are there any other alternatives to anticoagulation? Literature search was performed using the above questions in context on PubMed; the initial search populated seventy-five articles. After careful selection process and excluding duplicate entries and articles not answering the above question, the narrative review was written.

\section{Is over Anticoagulation a Problem?}

Bleeding related wound complications range from persistent wound leakage, superficial infection, deep infection and hematoma to PJI leading to major revision surgery. It is well documented that patients requiring therapeutic anticoagulation within the perioperative period have higher rates of bleeding-related complications [11] [12] [13]. A systematic review has reported up to two fold increase in bleeding complications in elderly patients who are on anti-coagulation [14]. Sachs et al. compared the complications of anticoagulation to prevent thromboembolic disease in patients with knee arthroplasty and found that the warfarin group had twice the infection rate as the control group (0.6\% vs. $0.3 \%)$ [13]. McDougall et al. highlighted a significant increase in risk of deep infection, wound hematoma, ooze, and superficial infection in patients undergoing total hip arthroplasty who require warfarin in the perioperative period [12]. The choice of anti-coagulant, however, is still controversial. There is also a lack of studies that look at complications in patients undergoing arthroplasty with therapeutic treatment with either warfarin plus or minus heparin and other combinations [12]. 


\section{What Is the Best Guidance on Use of Anticoagulant to Prevent and Treat VTE?}

Venous thromboembolism and appropriate selection of an anticoagulant remains a continuing concern in patients undergoing elective primary and revision TJA [7]. There has been a tremendous change in the past decade, in terms of guidelines put forward by the American Academy of Orthopaedic Surgeons (AAOS) and the American College of Chest Physicians (ACCP). However a lack of consensus still exists, as the guidelines do not recommend a preferred thromboprophylaxis or an optimal regimen [7] [15]. The balance of using a potent anticoagulant versus bleeding complications is difficult to achieve. Although more potent anticoagulants may successfully reduce venous thromboembolism, particularly asymptomatic events, their use may lead to increased bleeding, infection, and wound complications [7] [11]. This demands for risk stratification among patients. To our knowledge, there have been very few studies that have investigated the efficacy of potent anticoagulation, based on risk. Determining the high-risk patients is often subjective and difficult due to the paucity of studies that have elucidated the risk factors.

\section{Which Anticoagulant Drug Works Best?}

A multi-institutional study recently demonstrated that low-molecular-weight heparin and warfarin in higher-risk patients do not necessarily result in a reduction in the incidence of symptomatic venous thromboembolism. Aspirin administered to the higher-risk patients seems to be as effective as low-molecular weight heparin and warfarin [7]. Most surgeons use aspirin in only standard-risk to moderate-risk patients and use more potent anticoagulation for patients at increased risk, due to lack of studies evaluating the efficacy in high-risk group [7]. However, aspirin has been found to demonstrate efficacy in revision arthroplasty, which is considered to be a higher- risk procedure with increase morbidity compared to TJA [3]. Deirmengian et al. reported a lower rate of thromboembolic events in patients who received aspirin (0.56\%) compared with those who received warfarin (1.75\%), and aspirin also demonstrated a lower rate of bleeding events [7] [16]. Despite these reports there is no consensus, due to lack of a universally accepted risk stratification tool. Moreover use of potent anticoagulation and warfarin are associated with a higher rate of complications throughout the literature [2] [7] [10] [17].

\section{What Is the Risk of Recurrence?}

There is a lifelong risk of recurrence in patients with history of previous venous thrombroembolism [1]. These patients who have had a previous episode and need further surgery, revision for all causes including infection are considered to be high risk, however this risk has not been quantified [1]. The authors reported the relative risk of a VTE by 90 days in patients who had undergone a joint replacement with a history of VTE compared with those with a joint replacement 
and no history of VTE was 6.3 [1]. Despite using mechanical prophylaxis and anticoagulation, patients with previous history of VTE had a $3.4 \%$ chance of having a recurrence. In the same cohort there were complications due to bleeding from anticoagulation, some of which were more clinically significant than recurrent VTE. One patient who developed the deep infection remained infected despite a washout, and has been placed on long-term antibiotic suppression treatment [1]. D'Appuzzo et al. found evidence that the diagnosis of PE was associated with an increased risk of hematoma formation, post-operative infection, gastrointestinal bleeding and thrombocytopenia, indicating that anticoagulation has the potential to cause harm [18]. Anticoagulation increases the risk of complications and wound problems [1] [19].

\section{Is Recurrence of VTE Time Based Post-Operatively? What is the Ideal Duration of Treatment?}

The timing of symptomatic pulmonary embolism following TJA is controversial [4]. Studies have found that the vast majority of cases of pulmonary embolism (PE) occur early in the postoperative course, suggesting that extended prophylaxis may not be warranted [4] [20]. In 2006, Bjørnarå et al. reported that pulmonary emboli occurred at a median of 12 days following major joint arthroplasty [4] [6]. In 2007, Hope et al. found that the timing of PE varied significantly with the age of the patients: in patients younger than the age of 40 years, the majority of PEs occurred around day 3; however, in patients between the ages of 40 to 60 years, PEs tended to occur later, around day 11 [4] [20].

There is varying consensus with regard to the duration of postoperative anticoagulation in TJA. Given the risk of adverse events, it is desirable to limit the duration of anticoagulation to only the minimum duration required. Authors have reported that PE occurs early in the postoperative period. For longer prophylaxis protocols, the most aggressive anticoagulation may need to be employed for only the first two postoperative weeks [4].

\section{Are There Any Other Alternatives to Anticoagulation?}

Although anticoagulation is the preferred treatment of VTE, vena cava filters are important therapeutic options in the management of selected patients. This is when anticoagulation is contraindicated, its use causes complications, and its use is risk prone [21]. These classic indications have over the decades given way to extended indication with vena cava interruptions, achieved by percutaneous image guided insertion of an inferior vena cava (IVC) filter which has proved to be an important therapeutic option in management of selected patients with VTE [21].

\section{Conclusion}

Balanced anticoagulation is a difficult task to achieve, especially for patients undergoing complex arthroplasty surgery, including revision surgery. The potential consequences of over anticoagulation on the outcome of TJA and revision TJA 
are life changing and devastating. On the contrary falling short of adequate anticoagulation can be fatal leading to recurrent thromboembolic events. There is an urgent need for risk stratification tools to help surgeons and certainly the need for a multidisciplinary approach, as this is out of the scope of the operating surgeon alone. The indications of use of IVC filters are expanding, however their role in orthopedic surgery is still not clear. There is an urgent need for specialty specific guidelines for managing patient on anticoagulation undergoing TJA, and revision arthroplasty procedures.

\section{Conflicts of Interest}

The authors declare no conflicts of interest regarding the publication of this paper.

\section{References}

[1] Allen, D. and Sale, G. (2014) Lower Limb Joint Replacement in Patients with a History of Venous Thromboembolism. The Bone \& Joint Journal, 96-B, 1515-1519. https://doi.org/10.1302/0301-620X.96B11.33492

[2] Huang, R., Buckley, P.S., Scott, B., Parvizi, J. and Purtill, J.J. (2015) Administration of Aspirin as a Prophylaxis Agent against Venous Thromboembolism Results in Lower Incidence of Periprosthetic Joint Infection. Journal of Arthroplasty, 30, 39-41. https://doi.org/10.1016/j.arth.2015.07.001

[3] Huang, R.C., Parvizi, J., Hozack, W.J., Chen, A.F. and Austin, M.S. (2016) Aspirin Is as Effective as and Safer Than Warfarin for Patients at Higher Risk of Venous Thromboembolism Undergoing Total Joint Arthroplasty. Journal of Arthroplasty, 31, 83-86. https://doi.org/10.1016/j.arth.2016.02.074

[4] Parvizi, J., Huang, R., Raphael, I.J., Maltenfort, M.G., Arnold, W.V. and Rothman, R.H. (2015) Timing of Symptomatic Pulmonary Embolism with Warfarin Following Arthroplasty. Journal of Arthroplasty, 30, 1050-1053. https://doi.org/10.1016/j.arth.2015.01.004

[5] Gromov, K., Jørgensen, C.C., Petersen, P.B., Kjaersgaard-Andersen, P., Revald, P., Troelsen, A., et al. (2019) Complications and Readmissions Following Outpatient Total Hip and Knee Arthroplasty: A Prospective 2-Center Study with Matched Controls. Acta Orthopaedica, 90, 281-285. https://doi.org/10.1080/17453674.2019.1577049

[6] Bjørnarå, B.T., Gudmundsen, T.E. and Dahl, O.E. (2006) Frequency and Timing of Clinical Venous Thromboembolism after Major Joint Surgery. The Journal of Bone and Joint Surgery. British Volume, 88, 386-391. https://doi.org/10.1302/0301-620X.88B3.17207

[7] Tan, T.L., Foltz, C., Huang, R., Chen, A.F., Higuera, C., Siqueira, M., et al. (2019) Potent Anticoagulation Does Not Reduce Venous Thromboembolism in High-Risk Patients. The Journal of Bone and Joint Surgery. American Volume, 101, 589-599. https://doi.org/10.2106/JB/S.18.00335

[8] Sobti, D.A. and Sudhakar, M.J. (2017) High Body Mass Index and Its Effect on Total Knee Replacement. Journal of Arthritis, 6, 4. https://doi.org/10.4172/2167-7921.1000245

[9] Wagenaar, F.-C.B.M., Löwik, C.A.M., Zahar, A., Jutte, P.C., Gehrke, T. and Parvizi, J. (2019) Persistent Wound Drainage after Total Joint Arthroplasty: A Narrative Re- 
view. Journal of Arthroplasty, 34, 175-182.

https://doi.org/10.1016/j.arth.2018.08.034

[10] Patel, V.P., Walsh, M., Sehgal, B., Preston, C., DeWal, H. and Di Cesare, P.E. (2007) Factors Associated with Prolonged Wound Drainage after Primary Total Hip and Knee Arthroplasty. The Journal of Bone and Joint Surgery. American Volume, 89, 33-38. https://doi.org/10.2106/JBJS.F.00163

[11] Radovanovic, I., Queally, J., Bahari, S., Sproule, J. and McElwain, J. (2012) Anticoagulant Use and Its Effect on Bleeding and Complications in Total Knee Arthroplasty. Acta Orthopaedica Belgica, 78, 187-191.

[12] McDougall, C.J., Gray, H.S., Simpson, P.M., Whitehouse, S.L., Crawford, R.W. and Donnelly, W.J. (2013) Complications Related to Therapeutic Anticoagulation in Total Hip Arthroplasty. Journal of Arthroplasty, 28, 187-192. https://doi.org/10.1016/j.arth.2012.06.001

[13] Sachs, R.A., Smith, J.H., Kuney, M. and Paxton, L. (2003) Does Anticoagulation Do More Harm than Good? A Comparison of Patients Treated without Prophylaxis and Patients Treated with Low-Dose Warfarin after Total Knee Arthroplasty. Journal of Arthroplasty, 18, 389-395. https://doi.org/10.1016/S0883-5403(03)00071-8

[14] Hutten, B.A., Lensing, A.W., Kraaijenhagen, R.A. and Prins, M.H. (1999) Safety of Treatment with Oral Anticoagulants in the Elderly. A Systematic Review. Drugs Aging, 14, 303-312. https://doi.org/10.2165/00002512-199914040-00005

[15] Mont, M.A. and Jacobs, J.J. (2011) AAOS Clinical Practice Guideline: Preventing Venous Thromboembolic Disease in Patients Undergoing Elective Hip and Knee Arthroplasty. Journal of the American Academy of Orthopaedic Surgeons, 19, 777-778. https://doi.org/10.5435/00124635-201112000-00008

[16] Deirmengian, G.K., Heller, S., Smith, E.B., Maltenfort, M., Chen, A.F. and Parvizi, J. (2016) Aspirin Can Be Used as Prophylaxis for Prevention of Venous Thromboembolism after Revision Hip and Knee Arthroplasty. Journal of Arthroplasty, 31, 2237-2240. https://doi.org/10.1016/j.arth.2016.03.031

[17] Aquilina, A.L., Brunton, L.R., Whitehouse, M.R., Sullivan, N. and Blom, A.W. (2012) Direct Thrombin Inhibitor (DTI) vs. Aspirin in Primary Total Hip and Knee Replacement Using Wound Ooze as the Primary Outcome Measure. A Prospective Cohort Study. HIP International, 22, 22-27. https://doi.org/10.5301/HIP.2012.9058

[18] D’Apuzzo, M.R., Keller, T.C., Novicoff, W.M. and Browne, J.A. (2013) CT Pulmonary Angiography after Total Joint Arthroplasty: Overdiagnosis and Iatrogenic Harm? Clinical Orthopaedics and Related Research, 471, 2737-2742. https://doi.org/10.1007/s11999-013-3041-4

[19] Pirmohamed, M., James, S., Meakin, S., Green, C., Scott, A.K., Walley, T.J., et al. (2004) Adverse Drug Reactions as Cause of Admission to Hospital: Prospective Analysis of 18820 Patients. BMJ, 329, 15-19.

https://doi.org/10.1136/bmj.329.7456.15

[20] Hope, W.W., Demeter, B.L., Newcomb, W.L., Schmelzer, T.M., Schiffern, L.M., Heniford, B.T., et al. (2007) Postoperative Pulmonary Embolism: Timing, Diagnosis, Treatment, and Outcomes. The American Journal of Surgery, 194, 814-818. https://doi.org/10.1016/j.amjsurg.2007.08.014

[21] DeYoung, E. and Minocha, J. (2016) Inferior Vena Cava Filters: Guidelines, Best Practice, and Expanding Indications. Seminars in Interventional Radiology, 33, 65-70. https://doi.org/10.1055/s-0036-1581088 